\title{
Diferencias en el perfil de competencias socio-emocionales en estudiantes universitarios de diferentes ámbitos científicos
}

\section{Juan Luis Castejón, Ma Pilar Cantero, Nélida Pérez}

Departamento de Psicología Evolutiva y Didáctica, Universidad de Alicante

\section{España}

Juan Luis Castejón Costa. Universidad de Alicante. Carretera de San Vicente, s/n. 03080. Alicante. Spain. E-mail: jl.castejon@ua.es

(C) Education \& Psychology I+D+i and Editorial EOS (Spain) 


\section{Resumen}

Introducción. El presente trabajo tiene como objetivo principal establecer el perfil de competencias socio-emocionales característico de una muestra de estudiantes pertenecientes a cada uno de los grandes ámbitos académicos: ciencias jurídicas, ciencias sociales, educación, humanidades, ciencia y tecnología, y salud. Así como analizar las diferencias existentes entre estos ámbitos.

Método. El estudio se realizó sobre una muestra amplia $(\mathrm{N}=608)$ de estudiantes universitarios pertenecientes a 14 titulaciones de la Universidad de Alicante (España). Los instrumentos de evaluación utilizados incluyen diferentes medidas de inteligencia emocional como el TMMS y el EQ-i.

Resultados. Los resultados del análisis de varianza en un sentido entre los diferentes ámbitos científicos pusieron de manifiesto que aparecen diferencias significativas en la práctica totalidad de los aspectos de la Inteligencia Emocional, con excepción de la variable manejo del estrés.

Discusión. A partir de estos resultados se establecen implicaciones para el desarrollo de competencias genéricas de tipo socio-emocional en el marco del Espacio Europeo de Educación Superior.

Palabras clave: Inteligencia emocional, estudiantes universitarios, diferencias en el perfil de competencias socio-emocionales, implicaciones para el desarrollo de competencias genéricas.

Recibido: 10/05/08 Aceptación provisional: 13/05/08 Aceptación definitiva: 28/05/08 


\begin{abstract}
Introduction. The main objective of this paper is to establish the profile of socio-emotional competencies characteristic of a sample of students from each of the big academic areas in higher education: legal sciences, social sciences, education, humanities, science and technology, and health. An additional objective was to analyse differences between these fields.
\end{abstract}

Method. The study was carried out on a large sample $(\mathrm{N}=608)$ of university students from 14 different degree programs at the University of Alicante (Spain). Assessment was carried out using different measures of emotional intelligence, such as TMMS and EQ-i.

Results. Results of the analysis of variance in one sense among the different scientific fields revealed that significant differences appear in practically all aspects of Emotional Intelligence, except for the stress management variable.

Discussion. Based on these results, implications are drawn for developing generic socioemotional competencies in the framework of the European Space for Higher Education.

Key words: Emotional intelligence, university students, differences in the socio-emotional competency profile, implications for developing generic competencies

Received: 05/10/08 Initial Acceptance: 05/13/08_ Final Acceptance: 05/28/08 


\section{Introducción}

Actualmente podemos apreciar una nueva dirección a cerca de los estudios sobre la inteligencia hasta ahora no tenida en cuenta. Nos referimos al mundo de las competencias emocionales, olvidadas en la investigación científica, por el estudio de la razón dentro de la psicología cognitiva.

Muchas son las referencias y estudios que podemos encontrar en la literatura, sobre todo en este último siglo, sobre la inteligencia; no obstante, parece necesario llevar a cabo una delimitación entre la inteligencia académica y la denominada inteligencia no-académica, dentro de la que la inteligencia social, práctica y, sobre todo, la inteligencia emocional ocupan un lugar muy importante.

La inteligencia académica aparece tradicionalmente relacionada con la inteligencia general o analítica definida en términos psicométricos como CI (Sternberg, 2000; Sternberg 2003; Sternberg, Castejón, Prieto, Hautamäki y Grigorenko, 2001; Sternberg, Prieto y Castejón, 2000). La llamada inteligencia no-académica se ha considerado distinta a la inteligencia académica por dos razones; una, porque muestra un desarrollo evolutivo diferente (Matthews, Zeidner y Roberts, 2003); otra, porque parece explicar diferencias en la realización más allá del CI (Bowman, Markham y Roberts, 2001; Sternberg, Grigorenko y Bundy, 2001; Sternberg, Forsythe, Hedlund, et al., 2000) constituyendo por tanto un aspecto distinto de la inteligencia psicométrica tradicional de carácter general. Algo que, sin embargo, sigue estando en discusión por quienes, como Gottfredson (2003), defienden la existencia de un factor general de inteligencia ( $\mathrm{g}$ ) y argumentan en contra de la existencia de un factor de inteligencia práctica distinto de la inteligencia académica (g). La inteligencia social, por otra parte, es un constructo amplio cuyos límites son más difíciles de establecer y que en la literatura científica aparece ligada, unas veces a la inteligencia emocional (Bar-On, 2000; Goleman, 1998), otras a la inteligencia práctica (Hendlund y Sternberg, 2000). Bajo la denominación de inteligencia emocional se encuentran a su vez dos concepciones diferentes, sino contrapuestas (Bar-On, 2000; Mayer, Salovey y Caruso, 2000). Una conceptualización amplia, que considera la inteligencia emocional como una combinación de atributos estrechamente relacionados con la personalidad, distinta del CI, y relacionada con las competencias ligadas al logro académico y profesional (Bar-On, 2000; Goleman, 1995, 1998; McCrae, 2000); y otra conceptualización 
más restrictiva, en la que se considera como capacidad para percibir y entender información emocional (Mayer, Caruso y Salovey, 2000; Mayer, Caruso, Salovey y Sitarenios, 2003). Revisiones amplias y rigurosas del concepto de inteligencia emocional (Matthews, Zeidner y Roberts, 2003; Zeidner, Mathews y Roberts, 2004) plantean que la evidencia científica acerca del constructo de inteligencia emocional es aun escasa.

Además, tanto el tipo como el grado en que se produce un efecto de los componentes socio-emocionales en dominios académicos y profesionales distintos, puede depender del ámbito de que se trate; es necesario pues definir el carácter general o específico de los distintos aspectos de la inteligencia no-académica, con el objeto de identificar, bien factores generales comunes a los diferentes dominios, bien componentes concretos de la inteligencia social, emocional y práctica, relacionados con cada dominio o campo profesional (Boyatzis, Goleman y Rhee, 2000). Para ello se debe establecer un perfil de competencias socio-emocionales para cada campo científico-profesional y analizar las diferencias o semejanzas entre unos y otros perfiles.

La perspectiva actual de estudio de las competencias abarca tanto el ámbito académico (González y Wagenaar, 2003) como profesional (McClelland, 1999). Los trabajos sobre la inteligencia práctica en el mundo laboral y en la vida diaria, y sobre constructos similares como los de la inteligencia emocional, constituyen nuevos instrumentos para evaluar competencias necesarias en el medio profesional (Sternberg et al., 2000). El término competencia define un conjunto de habilidades comprometidas en la obtención de logros y en la resolución de problemas del ámbito personal o profesional (García, 2003; Le Boterf, 2001; LevyLeboyer, 1997), mientras que la inteligencia se refiere a los subcomponentes de estas habilidades presentes en las formas generales del pensamiento y entendimiento (Sternberg, et al., 2000).

Las habilidades comprometidas en la inteligencia emocional, social y práctica, una vez relacionadas con el desempeño en el ámbito personal, profesional y en la vida diaria en general, constituyen modelos de competencias (Boyatzis, 1999; Boyatzis, Goleman y Rhee, 2000; Sternberg, et al, 2000). Además, la mayor parte de las competencias profesionales identificadas como competencias clave para el desempeño profesional constituyen o están muy cercanas a los aspectos estudiados dentro de la inteligencia emocional. En una encuesta nacional a 
empleados americanos, se encontró que seis de las siete competencias consideradas clave para el éxito profesional formaban parte de la inteligencia emocional (Ayers y Stone, 1999; Goleman, 1998); a pesar de lo cual estas competencias no están incorporadas en la mayor parte de los programas universitarios (Boyatzis, et al. 1995; Echeverría, 2002). Aunque el desempeño profesional no parece ser predicho o explicado únicamente por estas competencias (Schmidt y Hunter, 1998), las competencias socioemocionales parecen tener un poder explicativo más allá de otras variables (Caruso y Wolfe, 2001; Goleman, 1998, 2001; Sternberg, et al., 2000). Estas competencias tienen también su efecto sobre la inserción laboral o la empleabilidad (Caruso y Wolfe, 2001; Fallows y Steven, 2000; y particularmente, Hettich, 2000).

En el ámbito de la enseñanza superior universitaria, se están desarrollando recientemente varias líneas de trabajo orientadas a la formación basada en competencias. La implementación de la Declaración de Bolonia de 1999, sobre el establecimiento del Espacio Europeo de Educación Superior, ha llevado a propuestas concretas de diseño y desarrollo de programas educativos y curricula universitarios basados en competencias, tales como las del proyecto Tuning Educational Structures in Europe (González y Wagenaar, 2003) en el que se establecen una serie de competencias genéricas, la mayor parte de las cuales coinciden con las competencias socioemocionales estudiadas bajo las denominaciones de inteligencia emocional, social y práctica. Tal y como se concluye en el proyecto quedan, sin embargo, cuestiones abiertas acerca de "si estas competencias son comunes o específicas, cómo identificarlas, cómo incorporarlas a los curricula universitarios o cómo desarrollarlas en los estudios de Grado".

Se trataría por tanto de avanzar con nuevas propuestas para la integración y desarrollo de estas competencias en los curricula de la educación superior, como las recogidas en el libro editado por Fallows y Steven (2000), y otras orientaciones surgidas en Estados Unidos y Canadá contenidas en Boyatzis, Cowen y Kolb (1995), y Boyatzis, Wheeler y Wright (2001). Existen además planes de estudio y curricula universitarios en los que se están implementando dichas competencias, como es el caso de varias universidades australianas (Nunan, George y McCausland, 2000), la Harvard Business School de la Universidad de Harvard (Jaeger, 2003; Prahalad y Hamel, 1990), en EEUU, o la Sheffield Hallam University, en Inglaterra, por citar algunas de ellas. 


\section{Método}

\section{Participantes}

La muestra total está compuesta por 608 estudiantes de la Universidad de Alicante, de los cuales 98 pertenecen al área de Ciencias Jurídicas, 171 al área de Ciencia Sociales, 101 al área de Educación, 66 al área de Humanidades, 160 al área de Ciencia y Tecnología, y por último 12 al área de Salud. El rango de edad de la muestra oscila entre 20 y 34 años, siendo la media de 24,2 años. El $52 \%$ de sujetos son mujeres y el $48 \%$ hombres.

Todos los estudiantes que participaron en la investigación estaban matriculados en el último curso de las 14 titulaciones pertenecientes a los ámbitos de conocimiento mencionados con anterioridad, lo que nos aseguraba la superación de casi todas las materias troncales de la titulación.

\section{Instrumentos}

Los instrumentos utilizados parten del modelo cognitivo de la Inteligencia Emocional desarrollado por los autores Salovey y Mayer (1990), y a partir del cual se han elaborado diferentes modalidades de evaluación, aportando diferentes pruebas de evaluación.

En este estudio se utiliza la escala de autoinforme denominada Trait Meta Mood Scale-24, adaptada al castellano por Fernández-Berrocal, Extremera y Ramos (2004) a partir de la desarrollada en 1990 por Mayer y Salovey. Dicha escala está compuesta por 24 ítems, valorados por una escala Likert teniendo en cuenta la puntuación 1 como "nada de acuerdo" y 5 como "totalmente de acuerdo", en la que se evalúan tres dimensiones de la escala original: Atención a los sentimientos, Claridad en los sentimientos y Regulación o reparación emocional. El TMMS-24 está formado por 24 enunciados, 8 por cada factor y su fiabilidad para cada componente es: Atención (.90), Claridad (.90) y Reparación (.86). Además presenta una fiabilidad test-retest adecuada (Fernández-Berrocal, et al., 2004).

Otro de los instrumentos empleados en la investigación ha sido el EQ-i de Bar-On (1997; adaptado al castellano durante el curso de esta investigación). Inventario sobre una amplia gama de habilidades emocionales y sociales compuesto por 5 factores generales, que se descomponen en un total de 15 subescalas: inteligencia intrapersonal, inteligencia interper- 
sonal, adaptación, gestión del estrés, y humor general. Además, el inventario incluye 4 indicadores de validez que miden el grado con que los sujetos responden al azar o distorsionan sus respuestas y cuyo objetivo es reducir el efecto de deseabilidad social e incrementar la seguridad de los resultados obtenidos. La fiabilidad de consistencia interna Alfa de Crombach obtenida en la muestra de estudiantes universitarios para cada una de las escalas es la siguiente: Inteligencia intrapersonal (.75), Inteligencia interpersonal (.77), Adaptabilidad (.84), Gestión del estrés $(.83)$ y Humor general (.88).

\section{Procedimiento}

El procedimiento general seguido en la realización de esta investigación se desarrolló de acuerdo con el planteamiento general en dos fases.

En una primera fase se contactó con los directores de Departamento o, en su defecto, docentes de aquellas titulaciones enmarcadas dentro de los diferentes ámbitos de actuación (área de ciencias jurídicas, área de ciencias sociales, área de educación, área de humanidades, área de ciencias y tecnología, y área de salud) con la finalidad de informarles a cerca de las líneas y objetivos del proyecto y contar con su consentimiento. De esta forma, se acordaron citas con aquellos profesores dispuestos a colaborar en el estudio. Tras esta primera reunión con el profesorado, se pasó a seleccionar la muestra de participantes siguiendo un procedimiento de muestro aleatorio estratificado proporcional al número de estudiantes matriculados en las titulaciones incluidas en cada uno de estos ámbitos y cuyos docentes nos prestaban colaboración. El estudio final se realizó sobre una muestra representativa de estudiantes matriculados en los últimos cursos pertenecientes a diversas titulaciones enmarcadas dentro de los ámbitos de conocimiento anteriormente mencionados. A continuación, se pasó a la preparación de los instrumentos de recogida de datos y a la elaboración tanto de una carta de presentación sobre el proyecto, como a una ficha personal con el objetivo de recabar información necesaria para facilitar de forma personal e individualizada los resultados a cada uno de los participantes.

La segunda fase de investigación, consistió en la aplicación de las pruebas seleccionadas para la recogida de datos sobre los diferentes aspectos de inteligencia socio-emocional. La aplicación de las pruebas se realizó durante el curso, en las aulas de clase y de acuerdo con las instrucciones contenidas en los correspondientes manuales; el tiempo empleado por el alum- 
nado en cumplimentar las pruebas abarcó alrededor de las dos horas de duración, utilizándose para ello una sesión única.

El orden seguido en la administración de pruebas fue: Primeramente se repartió la carta de presentación junto con la ficha de datos personales para que, a la vez que se les explicaba de forma oral la finalidad perseguida con el estudio, quedara constancia escrita por parte del estudiante de su conformidad para participar a través de la realización de pruebas y facilitación de datos personales. En este escrito el equipo investigador se comprometía a tratar cualquier dato estadísticamente como grupo y no a nivel particular, así como a facilitar de forma individualizada y una vez recopilada toda la información necesaria, los resultados obtenidos. A continuación, se procedió a la aplicación de las siguientes pruebas de evaluación de la inteligencia emocional: el TMMS-24 y el EQi de Bar-On, en este orden.

\section{Diseño y análisis estadístico}

El diseño empleado es de tipo ex post facto de comparación de grupos; mientras que como técnica de análisis estadístico se utiliza el análisis de varianza ANOVA de un sentido para identificar aquellas variables que contribuyen a diferenciar de forma estadísticamente significativa entre grupos. Las comparaciones post hoc se realizan con el método de Mínima Diferencia Significativa (MDS).

\section{Resultados}

A continuación se presentan los resultados de los análisis realizados sobre cada una de las competencias evaluadas, en función de cada tipo de análisis.

En la tabla 1, donde vienen representadas las medias por grupos en una escala que varía de 1 a 5, podemos ver cómo no aparecen grandes diferencias entre los distintos ámbitos educativos. No obstante analizando las tres variables del TMMS, en Atención a las emociones, por ejemplo, los estudiantes de CC y Tecnología obtienen la menor puntuación, frente a los estudiantes de Salud y Educación que alcanzan la puntuación más alta; tanto en Claridad como en Control emocional, es el grupo de CC Jurídicas el que queda por encima del resto, frente al de Salud que obtiene la menor puntuación. En cuanto a las variables del EQ-i, en la Intrapersonal sigue predominando el grupo de CC Jurídicas, frente al de Salud que continúa 
obteniendo la puntuación más baja; en la variable Interpersonal son los estudiantes de CC Sociales los que mayor puntúan en contraposición a los de Salud; en Manejo del estrés el área de CC y Tecnología predomina frente al de Humanidades que se sitúa a la cola; en Adaptabilidad así como en Estado de ánimo destacan los estudiantes pertenecientes a CC Jurídicas, mientras los de Salud siguen puntuando por debajo del resto; y para concluir, comentar que los resultados totales obtenidos en el EQ-i continúan atribuyendo las puntuaciones más altas a CC Jurídicas y las más bajas al área de Salud. Es en la variable Interpersonal del EQ-i donde todos los campos científicos obtienen mayores puntuaciones, a excepción de los estudiantes de CC Sociales que alcanzan su máxima puntuación en Estado de ánimo, variable también perteneciente al EQ-i.

Tabla 1. Medias de grupo para cada uno de los seis grupos científicos.

\begin{tabular}{ccccccc} 
& \multicolumn{5}{c}{ CAMPO CIENTÍFICO } \\
\cline { 2 - 7 } & 1 & 2 & 3 & 4 & 5 & 6 \\
\hline VARIABLES & CC Jurídicas & CC Sociales & Educación & Humanidades & CC y Tecnología & Salud \\
\hline Atención & 3.17 & 3.20 & 3.31 & 3.26 & 2.90 & 3.30 \\
Claridad & 3.57 & 3.34 & 3.30 & 3.44 & 3.37 & 3.00 \\
Control & 3.76 & 3.55 & 3.37 & 3.42 & 3.50 & 3.26 \\
Intrapersonal & 3.99 & 3.82 & 3.62 & 3.79 & 3.68 & 3.45 \\
Interpersonal & 4.40 & 4.45 & 4.38 & 4.25 & 4.15 & 4.08 \\
Manejo estrés & 3.68 & 3.57 & 3.57 & 3.47 & 3.71 & 3.57 \\
Adaptabilidad & 4.04 & 3.88 & 3.87 & 3.81 & 3.98 & 3.77 \\
Estado ánimo & 4.08 & 3.99 & 3.84 & 3.84 & 3.98 & 3.82 \\
EQI Total & 4.05 & 3.91 & 3.87 & 3.85 & 3.91 & 3.74 \\
\hline
\end{tabular}

En la gráfica 1 aparecen representados de forma más visible los resultados comentados con anterioridad. 


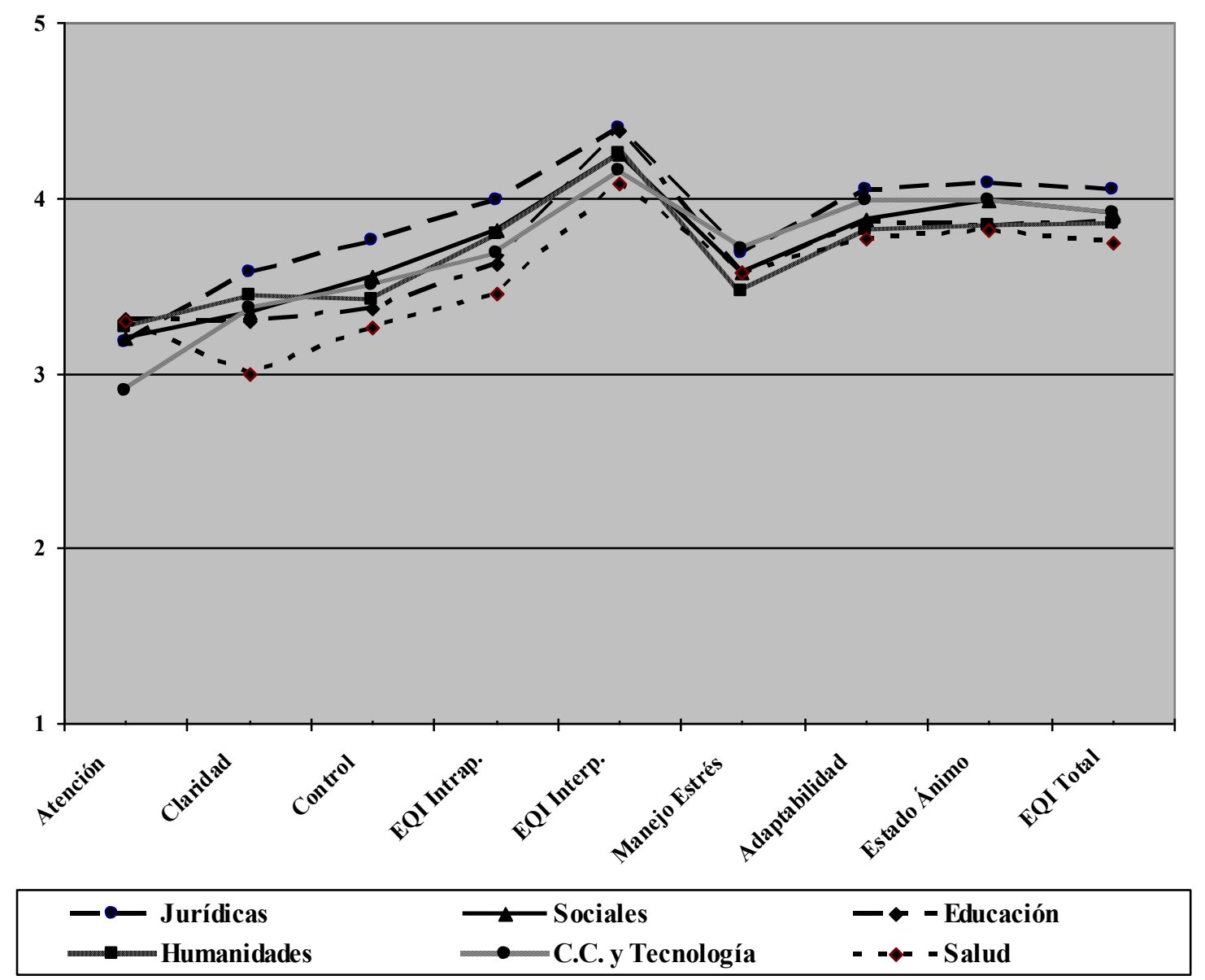

Gráfico 1. Representación de las medias de grupo por ámbitos científicos.

En la tabla 2, donde aparecen los resultados obtenidos en el análisis de varianza ANOVA de un sentido, vemos cómo aparecen diferencias significativas en todas las variables excepto en el Manejo de Estrés.

Tabla 2. Resultados del análisis de varianza, ANOVA en un sentido, realizado sobre las variables emocionales para establecer las diferencias entre grupos.

\begin{tabular}{cccccc}
\hline Variable & Suma de cuadrados & gl & Media cuadrática & F & p \\
\hline Atención & 13.252 & 5 & 2.650 & 4.799 & .000 \\
Claridad & 6.513 & 5 & 1.303 & 2.643 & .022 \\
Control & 9.592 & 5 & 1.918 & 3.453 & .004 \\
Intrapersonal & 9.734 & 5 & 1.947 & 6.622 & .000 \\
Interpersonal & 5.513 & 5 & 1.103 & 6.417 & .000 \\
Manejo Estrés & 3.693 & 5 & .739 & 1.330 & .250 \\
Adaptabilidad & 3.451 & 5 & .690 & 2.527 & .028 \\
Estado Ánimo & 4.223 & 5 & .845 & 2.866 & .014 \\
Total EQ-i & 2.722 & 5 & .544 & 4.243 & .001 \\
\hline
\end{tabular}


En la tabla 3, se ofrece un resumen de las diferencias encontradas en la prueba de comparaciones múltiples (MDS) para los factores del TMMS. Los resultados muestran que los estudiantes pertenecientes al ámbito científico de CC y Tecnología poseen una media significativamente menor en Atención emocional en comparación con los estudiantes de las áreas correspondientes a CC Jurídicas, CC Sociales, Educación y Humanidades. En la variable Claridad emocional, son los estudiantes de CC Jurídicas los que muestran una media significativamente mayor en relación a los estudiantes pertenecientes a las áreas de CC Sociales, Educación, CC y Tecnología y Salud; mientras que los estudiantes de Humanidades poseen una media significativamente mayor que los de Salud. En el último factor medido por el TMMS, es decir Control emocional, son los estudiantes de CC Jurídicas los que presentan una media significativamente mayor que el resto de ámbitos científicos.

Tabla 3. Resumen de las diferencias resultantes en la prueba de comparaciones múltiples para los factores del TMMS.

\begin{tabular}{|c|c|c|c|c|c|c|c|c|c|c|c|c|c|c|c|c|c|c|}
\hline \multirow[b]{3}{*}{ GRUPO } & \multicolumn{18}{|c|}{ VARIABLES } \\
\hline & \multicolumn{6}{|c|}{ Atención } & \multicolumn{6}{|c|}{ Claridad } & \multicolumn{6}{|c|}{ Control } \\
\hline & 1 & 2 & 3 & 4 & 5 & 6 & 1 & 2 & 3 & 4 & 5 & 6 & 1 & 2 & 3 & 4 & 5 & 6 \\
\hline 1 & --- & & & & & & --- & & & & & & --- & & & & & \\
\hline 2 & .809 & --- & & & & & $.008^{*}$ & --- & & & & & $.025^{*}$ & --- & & & & \\
\hline 3 & .213 & .245 & --- & & & & $.007^{*}$ & .679 & --- & & & & $.000^{*}$ & .059 & --- & & & \\
\hline 4 & .468 & .558 & .696 & --- & & & .225 & .328 & .222 & --- & & & $.005^{*}$ & .251 & .647 & --- & & \\
\hline 5 & $.005^{*}$ & $.000 *$ & $.000^{*}$ & $.001 *$ & --- & & $.027^{*}$ & .653 & .426 & .531 & --- & & $.006^{*}$ & .519 & .191 & .517 & --- & \\
\hline 6 & .572 & .634 & .988 & .855 & .075 & --- & $.008 *$ & .110 & .164 & $.048 *$ & .078 & --- & $.028 *$ & .196 & .631 & .484 & .293 & --- \\
\hline
\end{tabular}

1= CC Jurídicas. $\quad$ 2=CC Sociales. $\quad 3=$ =Educación. $\quad$ 4=Humanidades. $\quad$ 5=CC y Tecnología. $\quad 6=$ Salud.

Nota: Los números indican el valor de la probabilidad asociada a las diferencias entre pares de grupos.

$*$ Diferencias estadísticamente significativas entre dos grupos $(\mathrm{p}<.05)$ 
En la tabla 4, viene representado un resumen de las diferencias resultantes en la prueba de comparaciones múltiples MDS para los factores del EQ-i.

Tabla 4. Resumen de las diferencias resultantes en la prueba de comparaciones múltiples para los factores del EQ-i.

\begin{tabular}{|c|c|c|c|c|c|c|c|c|c|c|c|c|c|c|c|c|c|c|}
\hline \multirow[b]{3}{*}{ GRUPO } & \multicolumn{18}{|c|}{ VARIABLES } \\
\hline & \multicolumn{6}{|c|}{ Intrapersonal } & \multicolumn{6}{|c|}{ Interpersonal } & \multicolumn{6}{|c|}{ Manejo del Estrés } \\
\hline & 1 & 2 & 3 & 4 & 5 & 6 & 1 & 2 & 3 & 4 & 5 & 6 & 1 & 2 & 3 & 4 & 5 & 6 \\
\hline 1 & --- & & & & & & --- & & & & & & --- & & & & & \\
\hline 2 & $.011^{*}$ & --- & & & & & $.008^{*}$ & --- & & & & & .227 & --- & & & & \\
\hline 3 & $.000^{*}$ & $.006^{*}$ & --- & & & & .778 & $.019^{*}$ & --- & & & & .301 & .957 & --- & & & \\
\hline 4 & $.019^{*}$ & .735 & .057 & --- & & & $.027 *$ & .951 & .052 & --- & & & .069 & .361 & .378 & --- & & \\
\hline 5 & $.000^{*}$ & $.022 *$ & .461 & .156 & --- & & $.000^{*}$ & $.022 *$ & $.000^{*}$ & .090 & --- & & .822 & .104 & .172 & $.031 *$ & --- & \\
\hline 6 & $.002 *$ & $.033^{*}$ & .327 & .058 & .191 & --- & $.017^{*}$ & .184 & $.025^{*}$ & .213 & .617 & --- & .626 & .998 & .984 & .679 & .556 & --- \\
\hline
\end{tabular}

\begin{tabular}{|c|c|c|c|c|c|c|c|c|c|c|c|c|c|c|c|c|c|c|}
\hline \multirow[b]{3}{*}{ GRUPO } & \multicolumn{18}{|c|}{ VARIABLES } \\
\hline & \multicolumn{6}{|c|}{ Adaptabilidad } & \multicolumn{6}{|c|}{ Estado de Ánimo } & \multicolumn{6}{|c|}{ TOTAL } \\
\hline & 1 & 2 & 3 & 4 & 5 & 6 & 1 & 2 & 3 & 4 & 5 & 6 & 1 & 2 & 3 & 4 & 5 & 6 \\
\hline 1 & --- & & & & & & --- & & & & & & --- & & & & & \\
\hline 2 & $.020 *$ & --- & & & & & .169 & --- & & & & & $.002 *$ & --- & & & & \\
\hline 3 & $.027 *$ & .879 & --- & & & & $.002 *$ & $.034 *$ & --- & & & & $.000 *$ & .400 & --- & & & \\
\hline 4 & $.006^{*}$ & .331 & .444 & --- & & & $.006^{*}$ & .077 & .922 & --- & & & $.000 *$ & .273 & .744 & --- & & \\
\hline 6 & .101 & .471 & .519 & .799 & .189 & --- & .127 & .324 & .921 & .884 & .332 & --- & $.007 *$ & .142 & .273 & .362 & .134 & --- \\
\hline
\end{tabular}

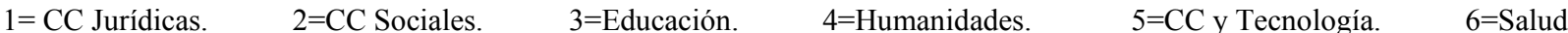

Nota: Los números indican el valor de la probabilidad asociada a las diferencias entre pares de grupos.

$*=$ Diferencias estadísticamente significativas entre dos grupos $(\mathrm{p}<.05)$. 
Para la primera variable, la Intrapersonal, los datos muestran cómo el grupo de CC Jurídicas posee una media significativamente mayor en comparación con el resto de ámbitos científicos estudiados; a la vez que el grupo de CC Sociales muestra una puntuación significativamente mayor en relación a los grupos de Educación, CC y Tecnología y Salud. En la variable Interpersonal, los estudiantes pertenecientes al ámbito de las CC Jurídicas son los que muestran una media significativamente, por un lado, mayor en comparación con los estudiantes de Humanidades, CC y Tecnología y Salud; y por otro, menor en relación al ámbito de las CC Sociales. Mientras que éste último, a su vez, presenta una media significativamente mayor en comparación a los grupos de Educación y CC y Tecnología. Por último, dentro de esta variable destacar la mayor puntuación que el ámbito Educativo presenta frente al CientíficoTecnológico y de la Salud. En cuanto a la variable Manejo del estrés, señalar cómo el grupo de Humanidades muestra una media significativamente menor en relación al ámbito Científico-Tecnológico. Para la variable Adaptabilidad a las emociones, son los estudiantes pertenecientes al ámbito de las CC Jurídicas los que poseen una media significativamente mayor en comparación con los estudiantes de CC Sociales, Educación y Humanidades; mientras que éstos últimos muestran, a su vez, una media significativamente menor en relación al grupo Científico-Tecnológico. En la variable Estado de ánimo, el grupo de CC Jurídicas es el que presenta unos resultados significativamente mayores en comparación con los grupos de Humanidades y Educación; mientras que éste último muestra una menor puntuación en relación con CC Sociales, así como éstos estudiantes con los de CC y Tecnología. Para finalizar, los resultados de la variable EQ-i Total nos muestran una media significativamente superior de los estudiantes pertenecientes al ámbito de las CC Jurídicas frente al resto de las áreas estudiadas.

\section{Discusión y conclusiones}

Tomados en conjunto los resultados obtenidos ponen de manifiesto que existen diferencias entre grupos de estudiantes universitarios pertenecientes a diversos ámbitos científicoprofesionales en las puntuaciones obtenidas en diferentes aspectos de la inteligencia emocional.

De esta forma vemos cómo el área de ciencias jurídicas permanece por encima del resto de ámbitos profesionales en todas las variables estudiadas, con excepción del manejo de 
estrés donde no se observan diferencias significativas en ninguna de las titulaciones. Y es que, tal y como apunta Relly (2005) para llegar a ser un abogado eficaz uno debe aprender a desarrollar determinados aspectos de la inteligencia emocional, destacando las competencias interpersonales como claves a la hora de mejorar las habilidades de negociación. También Fisher, Ury y Patton (1991) hacen una observación similar cuando escriben que en una negociación jurídica los sentimientos pueden llegar a resultar mucho más importantes que la palabra; y es que desmitificar la complejidad de las emociones puede llegar a ser una manera de explicar y empezar a comprender el papel tan importante que las competencias socio-emocionales pueden alcanzar a la hora de resolver los conflictos.

Por el contrario, son los estudiantes de ciencias de la salud los que puntúan por debajo en prácticamente todas las variables, salvo en el manejo de estrés y el cociente emocional total; resultados que pueden ser debidos a la reducida muestra de participantes que, en la actualidad, ya se ha conseguido ampliar sensiblemente. Así, Baños y Pérez (2005) destacan la importancia de las competencias genéricas o transversales de tipo socio-emocional en la formación del graduado universitario y, más específicamente, para los futuros profesionales de la salud. Tradicionalmente en las titulaciones de esta área la atención se ha centrado en las competencias específicas y ha obviado la preocupación por la instauración de ciertas competencias transversales, a pesar de ser consideradas como imprescindibles. Pero es en los últimos años cuando se ha realizado un notable esfuerzo para alcanzar un reconocimiento más o menos unánime en lo que debe ser competente un profesional de la salud. Se destaca así las competencias transversales interpersonales como un elemento de importancia excepcional en la formación universitaria de cualquier titulación y, por supuesto, en ciencias de la salud; donde la capacidad para trabajar en equipo es un componente esencial.

En cuanto al área educativa, a veces se piensa de forma errónea que las competencias afectivas y emocionales no son imprescindibles en el profesorado; sin embargo la inteligencia emocional conlleva el desarrollo de un conjunto de habilidades que todo docente debería aprender por dos razones: porque las aulas son el modelo de aprendizaje socio-emocional de mayor impacto para los alumnos, y porque la investigación está demostrando que unos adecuados niveles de inteligencia emocional ayudan a afrontar con mayor éxito los contratiempos cotidianos a los que el profesorado se enfrenta (Extremera y Fernández-Berrocal, 2004). De esta forma, educar la inteligencia emocional se convierte en una tarea necesaria en el ámbito 
educativo, puesto que llegar a dominar estas habilidades resulta primordial para el desarrollo evolutivo y socio-emocional del alumnado.

También desde hace varios años se viene planteando, desde el ámbito de las ciencias sociales, la importancia de que quien ocupa un puesto de dirección en una organización ejerza también liderazgo. Y es que el enfoque de la inteligencia emocional está teniendo cada vez mayor repercusión en el ámbito empresarial (Cooper y Sawaf, 1998; Goleman, 1999; Weisinger, 1998), así como su relación con el liderazgo (Senge, 1992). Goleman, por ejemplo, cita el estudio de Boyatzis en dos mil supervisores, gerentes y ejecutivos de diferentes empresas, encontrando que había 16 habilidades compartidas por quienes eran considerados "estrellas", de las cuales todas excepto 2 eran de inteligencia emocional. Otros estudios como el de Quintillán (2005) muestran cómo los estudiantes se autoperciben con un nivel medio-alto de características de liderazgo eficaz, encontrándose los mejores resultados en las habilidades interpersonales. Se destaca, además, que un nivel de liderazgo eficaz medio-alto es, sin duda, satisfactorio por lo que sería interesante indagar la incidencia de la formación que brinda la universidad respecto a esta competencia considerada, tanto por el alumnado como por el profesorado, como fundamental en el futuro empresario.

En cuanto a los estudiantes de ciencia y tecnología señalar el bajo grado de atención emocional que mantienen en relación al resto de ámbitos académicos; lo que resulta beneficioso ya que demasiada atención a los sentimientos puede afectar al rendimiento. En la actualidad, muchas de las titulaciones tecnológicas siguen manteniendo una distancia importante entre el perfil de egresado que forma la universidad y el perfil profesional que demanda la sociedad. Pero como bien dice Juan et al. 2006, las competencias genéricas o transversales juegan un papel importantísimo en la formación del ingeniero y es que "constituyen el cemento que une todas sus competencias específicas generando una sinergia que establecerá la diferencia entre el saber y el saber aplicar de manera óptima y con deontología profesional". Para un ingeniero las competencias interpersonales juegan así un papel fundamental ya que desarrollan su capacidad de organizar y estructurar el trabajo, mucho más cuando de él se espera la dirección de grupos.

Como hemos observado, educar la inteligencia emocional de los estudiantes se está convirtiendo en una tarea necesaria en el ámbito universitario y la mayoría de docentes considera primordial el dominio de estas habilidades para el desarrollo evolutivo y socio-emocional 
del alumnado. No obstante, aunque las representaciones mentales de los profesores sobre la incorporación de dichas competencias genéricas en la formación universitaria valora la importancia que éstas tienen en el perfil de formación, queda patente su reticencia a incorporarlas en el propio currículum académico, hecho atribuible a posibles carencias en su preparación docente (Corominas et al., 2006).

La actividad docente que realiza la universidad tiene como intención y resultado la formación intelectual de los ciudadanos y la preparación de profesionales para su posterior incorporación al mercado de trabajo (Gallardo, 2006; Goñi, 2000). Pero tal incorporación, unida a la formación teórica que se suele dar en la universidad, hace que los estudiantes tengan que formarse por su cuenta en el difícil tránsito a ese ámbito laboral.

Esta sociedad, que está en continua transformación, demanda una adaptación de la universidad y al mismo tiempo una adecuación de la formación universitaria a las expectativas de la demanda social y del mercado laboral. En este sentido se pronuncia Amador (1996) cuando afirma que en el binomio formación-empleo se debe dar una correspondencia entre la formación que requiere un puesto de trabajo y la formación que debe aportar un centro universitario, además, el marco curricular de cada titulación debe tener en cuenta el perfil profesional que pretende cada ciclo formativo. Los resultados ofrecidos por el estudio de González (2006) estiman cómo los estudiantes universitarios consideran necesario el desarrollo de competencias ligadas a la consecución del empleo para el que se les está formando.

Una de las vías para dar respuesta a la amplia gama de exigencias que han de afrontar los graduados universitarios en la sociedad del conocimiento que se está construyendo en Europa consiste en el estudio y desarrollo de las Competencias Genéricas y Específicas de los estudiantes, tanto en su preparación de base en niveles educativos previos como durante la realización de su formación universitaria (Lucas, 2007).

En coherencia con los resultados obtenidos, y de acuerdo con De Miguel et al. (2003), se constata que la evaluación del grado de satisfacción que tienen los profesionales, desde su visión práctica, sobre la formación universitaria puede ser efectuada desde la planificación y el desarrollo de los procesos de aprendizaje y la adecuación del perfil formativo de las titulaciones. Igualmente, de acuerdo con De la Fuente et al. (2004), el docente, el alumno o el profesional, puede delimitar rápidamente las competencias, en términos de contenidos de aprendizaje propuestos y de las propias actividades de aprendizaje, diseñadas para su construcción. 
Como muy acertadamente incidían García-Montalvo y Peiró (2001, p.28) "los procesos de inserción laboral tienen consecuencias importantes para los jóvenes, porque en parte determinan su carrera profesional, sus oportunidades de desarrollo personal y su bienestar psicológico".

La mayor parte del profesorado coincide en que el liderazgo docente del profesor universitario se consigue con un adecuado conocimiento personal, una alta autoestima, un control emocional destacado, así como un proyecto personal y docente motivador. Conseguir el éxito de los estudiantes, esto es, conseguir que voluntariamente desarrollen sus competencias científicas, profesionales e investigadoras a través de sus propias competencias socioemocionales, determinará sin duda un alto nivel de liderazgo docente y un aumento en la autoestima del profesorado universitario (Hué, 2007).

Un currículo por competencias profesionales que articula conocimientos globales, conocimientos profesionales y experiencias laborales, se propone reconocer las necesidades y problemas de la realidad. Tales necesidades y problemas se definen mediante el diagnóstico de las experiencias de la realidad social, de la práctica de las profesiones, del desarrollo de la disciplina y del mercado laboral y de la propia misión de la institución. Esta combinación de elementos permite identificar las necesidades hacia las cuales se orientará la formación profesional, de donde se desprenderá también la identificación de las competencias profesionales integrales o genéricas, indispensables para el establecimiento del perfil de egreso del futuro profesional. A pesar de que la formación personal, la educación para la producción y la formación social sean tres ejes relevantes para el acto educativo, por lo general, al definir las competencias de egreso en la educación superior se tiende a priorizar las competencias laborales centradas en el eje de educación para la producción (Larraín y González, 2005).

Si partimos de la consideración de que, por un lado, las habilidades comprometidas en la inteligencia emocional una vez relacionadas con el desempeño en el ámbito personal, profesional y en la vida diaria en general, constituyen modelos de competencias (Boyatzis, 1999; Boyatzis, Goleman y Rhee, 2000; Hedlund y Sternberg, 2000); y por otro lado, los modelos de inteligencia emocional incluyen competencias amplias de carácter socio-emocional (Mayer, Salovey y Caruso, 2000), podemos apreciar la importancia que tiene la identificación, evaluación y el desarrollo de estas competencias emocionales en el contexto educativo de la enseñanza superior universitaria. 
Cabe destacar, así, la importancia con la que hoy en día cuenta la inteligencia interpersonal dentro de la formación universitaria, ya que se trata de una de las competencias transversales más valoradas en el proyecto Tuning (González \& Wagenaar, 2003), razón por la que los estudiantes pertenecientes al ámbito científico no deben descuidar dicha faceta en su perfil de competencias. En este sentido parece necesario introducir dentro de la formación del estudiante métodos de enseñanza capaces de favorecer el desarrollo de la inteligencia interpersonal, tales como el trabajo cooperativo, las dinámicas de grupos y otros. Metodologías que pretenden mostrar cómo determinados objetivos tanto educativos como sociales se alcanzan coordinando acciones que, de otra manera, no se podrían conseguir; es decir, cómo del resultado del trabajo cooperativo se obtienen beneficios buscados y compartidos entre todos. Son numerosos los estudios que demuestran la superioridad del aprendizaje cooperativo sobre la competición y la individualidad en todos los niveles (Johnson, Maruyana, Johnson, Nelson y Skon, 1981). Así, Chickering y Gamson (1991) proponen como uno de los principios básicos para lograr un aprendizaje óptimo entre los universitarios fomentar la cooperación entre los estudiantes utilizando el aprendizaje cooperativo. De ahí la importancia y necesidad de formar al profesorado en esta innovadora metodología.

\section{Referencias}

Amador, F. (1996). Análisis de la formación universitaria en las Ciencias del Deporte. Su adecuación a los perfiles profesionales. Disponible en http://www.iusport. es/OPINION/amador98.htm.

Ayers, D. y Stone, B. (1999). Extension organization of the future: Linking emotional intelligence and core competencies. Journal of Extension, 37 (6). Disponible en http:/joe.org/joe/1999december/iw4.html.

Baños, J. E. y Pérez, J. (2005). Cómo fomentar las competencias transversales en los estudios de Ciencias de la Salud: una propuesta de actividades. Educación Médica, 8 (4), 216225.

Bar-On, R. (1997). BarOn Emotional Quotient Inventory Technical Manual. Toronto: MultiHealth Systems. 
Bar-On, R. (2000). Emotional and social intelligence: Insights from the Emotional Quotient Inventory (EQ-i). En R. Bar-On y J.D.A. Parker (Eds.). The handbook of emotional intelligence: Theory, development, assessment, and application at home, school, and in the workplace. (pp. 363-387). San Francisco, CA: Jossey-Bass Inc.

Bowman, D., Markham, P., y Roberts, R. (2001). Expanding the frontier of cognitive abilities: So much more than (plain) g! Learning and Individual Differences, 13(2), 127-158.

Boyatzis, R.E. (1999). Self-directed change and learning as a necesary meta-competency for success and effectiveness in the $21^{\text {st }}$ century. En R. Sims \& J.G. Veres (Eds.), Keys to employee success in the coming decades (pp. 15-32). Westport: Greenwood.

Boyatzis, R., Goleman, D., y Rhee, K. (2000). Clustering competence in emotional intelligence. Insights from the emotional competence inventory (ECI). En R. Bar-On \& J.D.A. Parker (Eds.), Handbook of emotional intelligence (pp. 343-367). San Francisco: Jossey-Bass.

Boyatzis, R.E., Cowen, S.S., y Kolb, D.A. (Eds.), (1995). Innovation in professional education: Steps on a journey from teaching to learning. San Francisco: Jossey-Bass.

Boyatzis, R.E., Wheeler, J., y Wright, R. (2001). Competency development in graduate education: A longitudinal perspective. En Proceedings of the First World Conference on Self-Directed Learning. Montreal: GIRAT.

Caruso, D.R., y Wolfe, Ch.J. (2001). Emotional intelligence in the workplace. En J., Ciarrochi, J. Forgas y J.D. Mayer (Eds.), Emotional intelligence in everyday life. Londres: Psychology Press.

Chickering, A.W. y Gamson, Z.F. (1991). Applying the seven principles for good practice in undergraduate education. New Directions for Teaching and Learning, 47, 34-67.

Cooper, R. y Sawaf, A. (1998). La inteligencia emocional aplicada al liderazgo y a las organizaciones. Bogotá: Norma.

Corominas, E., Tesouro, M., Capell, D., Teixidó, J., Pèlach, J. y Cortada, R. (2006). Percepciones del profesorado ante la incorporación de las competencias genéricas en la formación universitaria. Revista de Educación, 341, 301-336.

De la Fuente, J. (Cood.), (2004). Guía metodológica para la evaluación de la construcción del conocimiento profesional, promovido desde las titulaciones oficiales. Proyecto UCUA. UAL-11. Córdoba: UCUA.

De Miguel, M. (Coord.), (2003). Evaluación de la calidad de las titulaciones universitarias. Madrid: Ministerio de Educación, Cultura y Deporte. 
Echeverría, B. (2002). Gestión de la competencia de acción profesional. Revista de Investigación Educativa, 20(1), 7-42.

Extremera, N. y Fernández-Berrocal, P. (2004). La importancia de desarrollar la inteligencia emocional en el profesorado. Revista Iberoamericana de Educación, 33, 1-10.

Fallows, S., y Steven, C. (Eds.), (2000). Integrating key skills in higher education: employability, transferable skills and learning for life. Londres: Kogan Page.

Fernández-Berrocal, P., Extremera, N., y Ramos, N. (2004). Validity and reliability of the Spanish modified version of the Trait Meta-Mood Scale (TMMS-24). Psychological Report, 94, 751-755.

Fisher, R., Ury, W.y Patton, B. (1991). Getting to YES: Negotiations agreement without giving in, 2nd Edition. Random House Business Books.

Gallardo, M. Á. (2006). Evaluación de las competencias profesionales para la inserción laboral de los maestros de Educación Física. Electronic Journal of Research in Educational Psychology, 4 (3), 469-492. Obtenido el 27 de febrero de 2008 en http://www.investigacion-psicopedagogica.org/revista/articulos/10/espannol/Art_10_1 48.pdf

García, M. (2003). Entrenamiento en competencias. Encuentros en Psicología Social, 1(3), 27-32.

García-Montalvo, J. y Peiró, J.M. (2001). Capital Humano: El mercado laboral de los jóvenes. Formación, transición y empleo. Valencia: Instituto Valenciano de Investigaciones Económicas/Bancaja.

Goleman, D. (1995). Emotional Intelligence. New York: Bantam Books, Inc.

Goleman, D. (1998). Working with emotional intelligence. Santa Ana: Books on Tape, Inc.

Goleman, D. (1999). La inteligencia emocional en la empresa. Buenos Aires, Vergara.

Goleman, D. (2001). Emotional intelligence perspectives on a theory of performance. En C. Cherniss, \& D. Goleman (Eds.), The emotionally intelligent workplace. San Francisco: Jossey-Bass.

González, I. (2006). Dimensiones de evaluación de la calidad universitaria en el Espacio Europeo de Educación Superior. Electronic Journal of Research in Educational Psychology, 4 (3), 445-468. Obtenido el 27 de febrero de 2008 en http://www .investigacionpsicopedagogica.org/revista/articulos/10/espannol/Art_10_134.pdf

González, J., \& Wagenaar, R. (2003). Tuning Educational Structures in Europe. Final Report. Phase One. Bilbao: Universidad de Deusto. 
Goñi, A. (2000). Inserción laboral desde la Universidad. Vitoria: Vicerrectorado del Campus de Álava de la Universidad del País Vasco.

Gottfredson, L. (2003). Dissecting practical intelligence theory: Its claims and evidence. Intelligence, 31(4), 343-397.

Hendlund, J. y Sternberg, RJ. (2000). Too many intelligences? Integrating social, emotional and practical intelligence. En R. Bar-On y J.D.A. Parker (Eds.). The handbook of emotional intelligence (pp. 136-168). San Francisco: Jorsey-Bass.

Hettich, P. (2000). Transition processes from college to career. Washington: American Psychological Association.

Hué, C. (2007). Una experiencia de formación en competencias emocionales del profesorado universitario. En Innovación docente, tecnologías de la información y la comunicación e investigación educativa. Caminado hacia Europa. Zaragoza: Universidad de Zaragoza.

Jaeger, A. (2003). Job competencies and the curriculum: An inquiry into emotional intelligence in graduate professional education. Research in Higher Education, 44(6), 615639.

Johnson, D.W., Maruyama, G., Johnson, R., Nelson, D. y Skon, L. (1981). The effects of cooperative, competitive, and individualistic goal structures on achievement: A metaanalysis. Psychological Bulletin, 89, 47-62.

Juan, A., Andrés, J., Nieto, C., Suárez, M., Pérez, R., Cernuda, A., Luengo, C., Martínez, B., Riesco, M., Lanvín, D., Labra, J., Fondón, M. y Redondo, J.M. (2006). Definición de competencias específicas y genéricas del ingeniero en informática. En Docencia Universitaria Proyectos de Innovación Docente. Universidad de Oviedo.

Larraín A.M. y González L.E. (2005). Formación universitaria por competencias. Chile.

Le Boterf, G. (2001). Ingeniería de las competencias. Barcelona: Gestión 2000.

Levy-Leboyer, C. (1997). Gestión de las competencias: cómo analizarlas, cómo evaluarlas, cómo desarrollarlas. Barcelona: Gestión 2000.

Lucas, S. (2007). Desarrollo de competencias desde la enseñanza universitaria. Armonización con la Educación Secundaria y el mercado de trabajo, desde la Psicología Social de la Educación. Electronic Journal of Research in Educational Psychology, 5 (1), 125-158. Obtenido el 27 de febrero de 2008 en http://www.investigacionpsicopedagogica.org/revista/articulos/11/espannol/Art_11_161.pdf

Matthews, G., Zeidner, M., y Roberts, R. (2003). Emotional intelligence: Science and Myth. Cambridge: MIT Press. 
Mayer, J., Caruso, DR. y Salovey, P. (2000). Selecting a measure of emotional intelligence: The case of ability scales. En R. Bar-On y J. Parkes (Eds.). The handbook of emotional intelligence: Theory, development, assessment, and application at home, school, and in the workplace (pp. 320-342). San Francisco: Jossey-Bass/Pfeiffer.

Mayer, J., Caruso, DR., Salovey, P. y Sitarenios, G. (2003). Measuring emotional intelligence with the MSCEIT V2.0. Emotion, 3(1), 97-105.

Mayer, J., Salovey, P. y Caruso, D. (2000). Emotional intelligence as Zeitgeist, as personality, and as mental ability. En R.J. Sternberg (Ed.), Handbook of emotional intelligence (pp. 92-117). San Francisco: Jossey Bass.

McClelland, D.C. (1999). A guide to job competency assessment. Boston: McBer.

McCrae, R.R. (2000). Trait psychology and the revival of personality and culture studies. American Behavioral Scientist, 44 (1), 10-31.

Nunan, T., George, R. y McCausland, H. (2000). Implementing graduate skill at an Australian university. En S. Fallows \& C. Steven (Eds.), Integrating key skills in higher education: employability, transferable skills and learning for life. Londres: Kogan Page.

Prahalad, C.K. \& Hamel, G. (1990). The core competence of the Corporation. Harvard Business Review, Mayo, 79-92.

Quintillán, I. (2005). Liderazgo e inteligencia emocional: la formación y el perfil de los estudiantes del último año de Licenciatura en Dirección de Empresas. Revista Electrónica de la Facultad de Ciencias Empresariales de la Universidad Católica del Uruguay, 6.

Really, P. (2005). Teaching law students how to feel: using negotiations training to increase emotional intelligence. Negotiation Journal, 21 (2), 301-314.

Salovey, P. y Mayer, J.D. (1990). Emotional intelligence. Imagination, Cognition, and Personality, 9, 185-211.

Schmidt, F.L. y Hunter, J.E. (1998). The validity and utility of selection methods in personal psychology: Practical and theoretical implications of 85 years of research findings. Psychological Bulletin, 124, 262-274.

Senge, P. (1992). La quinta disciplina. Cómo impulsar el aprendizaje en la organización inteligente. Barcelona, Granica.

Sternberg, R.J. (2000). The concept of intelligence. En R.J. Sternberg (Ed.), Handbook of intelligence (pp. 3-15). Nueva York: Cambridge University Press.

Sternberg, R.J. (2003a). Reply to the book review on Practical Intelligence in Everyday Life. Intelligence, 31(4), 395-408. 
Sternberg, R.J. (2003b). Issues in the theory and measurement of successful intelligence: A reply to Body. Intelligence, 31, 331-337.

Sternberg, R.J., Castejón, J.L., Prieto, M.D., Hautamäki, J., y Grigorenko, E. (2001). Confirmatory factor analysis of the Sternberg Triarchic Abilities Test in Three International Samples: An empirical test of the Triarchic Theory. European Journal of Psychological Assessment, 17, 1-16.

Sternberg, R.J, Forsythe, G.B., Hedlund, J., Horvath, J.A., Wagner, R.K., Williams, W.M., Snook, A., y Grigorenko, E.L. (2000). Practical intelligence in everyday life. Nueva York: Cambridge University Press.

Sternberg, R.J., Grigorenko, E.L., y Bundy, D.A. (2001). The predictive value of IQ. MerrillPalmer Quarterly, 47(1), 1-41.

Sternberg, R.J., Prieto, M.D., y Castejón, J.L. (2000). Análisis factorial confirmatorio del Sternberg Triarchic Abilities Test (nivel H) en una muestra española: resultados preliminares. Psicothema, 12(4), 642-647.

Weisinger, H. (1998). La inteligencia emocional en el trabajo. Buenos Aires, Vergara.

Zeidner, M., Matthews, G., Roberts, R.D. (2004). Emotional intelligence in the workplace: A critical review. Applied Psychology. An International Review, 53(3), 371-399. 